\title{
Alleviating the Sparsity Problem in Collaborative Filtering by using an Adapted Distance and a Graph-based Method
}

\author{
Beau Piccart, Jan Struyf $f^{\dagger}$ Hendrik Blockeel ${ }^{\ddagger}$
}

\begin{abstract}
Collaborative filtering $(\mathrm{CF})$ is the process of predicting a user's interest in various items, such as books or movies, based on taste information, typically expressed in the form of item ratings, from many other users. One of the key issues in collaborative filtering is how to deal with data sparsity; most users rate only a small number of items.

This paper's first contribution is a distance measure. This distance measure is probability-based and is adapted for use with sparse data; it can be used with for instance a nearest neighbor method, or in graph-based methods to label the edges of the graph. Our second contribution is a novel probabilistic graph-based collaborative filtering algorithm called PGBCF that employs that distance. By propagating probabilistic predictions through the user graph, $\mathrm{PGBCF}$ does not only use ratings of direct neighbors, but can also exploit the information available for indirect neighbors. Experiments show that both the adapted distance measure and the graph-based collaborative filtering algorithm lead to more accurate predictions.
\end{abstract}

\section{Introduction}

Recommender systems [1] are software systems designed to propose, given a large set of available items (books, movies, music pieces, news articles, ...), the items that are most likely of interest to the user. Two common classes of algorithms used in recommender systems are content-based algorithms and collaborative filtering (CF) algorithms. Content-based algorithms base their recommendations on the content of the items (e.g., the text of a news article), while collaborative filtering algorithms base their recommendations on information recorded by the system about the preferences of other users.

Collaborative filtering systems may rely on ratings that users have explicitly assigned to (a subset of) the items, or on binary data, which records for each user the items that he or she has previously bought (e.g., in the context of an e-commerce system). This paper considers the former setting. Here, the input data can be represented in terms of a ratings matrix, which has one row for each user and one column for each item and in which the elements indicate the ratings given by the users to the various items. We assume that a rating is a

\footnotetext{
*beau.piccart@cs.kuleuven.be

†jan.struyf@cs.kuleuven.be

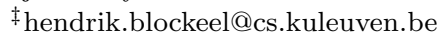

natural number taken from a fixed range (e.g., 1...5). If there are $m$ users and $n$ items, then the ratings matrix $A$ is an $m$ by $n$ matrix and the element $A_{i, j}$ is either the rating assigned by user $i$ to item $j$, or it is equal to the missing value indicator if user $i$ has not rated item $j$.

Given the ratings matrix, a collaborative filtering algorithm can recommend a number of items to a particular user by first predicting ratings for all items that the user has not rated yet, and by subsequently proposing the items with a high predicted rating. For example, to predict user $i$ 's rating for item $j$, a $k$-nearest neighbor based CI algorithm would consider the set of all other users who rated item $j$, among these search for the $k$ most similar ones (in terms of their other ratings), and subsequently take the most frequently occurring value among the neighbors' ratings for item $j$ as prediction. If predicted ratings are allowed to be real numbers, it may alternatively compute the mean instead of the most frequent value.

One of the most important issues in collaborative filtering is how to deal with data sparsity [3-6, 8, 9, 11]. Typically, users only rate a small percentage of the items and the ratings matrix is very sparse (includes many missing values). Data sparsity may be detrimental to accuracy because of the following two reasons. First, reliably estimating the similarity between two users becomes difficult because, due to the many missing values, there may be few items that are rated by both (and hence contain some information regarding their similarity). Second, to predict a rating $A_{i, j}$, one needs sufficient users different from user $i$ who also rated item $j$. Because of data sparsity there will be a relatively small number of such users resulting in a small input set for nearest neighbor.

This paper proposes an approach that deals with unknown values by switching to a probabilistic representation of the problem. That is, instead of working with ratings, our algorithms work with probability distributions over the domain of possible ratings. If a user's rating for an item is known, then the corresponding probability distribution has zero variance and places all probability mass on that particular rating. If it is 
unknown, then the algorithms make use of a uniform, global, or predicted distribution.

On top of this probabilistic representation, we propose an estimated distance measure to compute the similarity between the rating profiles of two users. This measure can be used in nearest neighbor style collaborative filtering algorithms. As we will see, this greatly improves the performance of the nearest neighbor $(\mathrm{kNN})$ approach.

Our second approach to improve upon the kNN approach consists of a graph-based algorithm called probabilistic graph-based collaborative filtering (PGBCF). This algorithm works on a user graph in which the users are nodes and the edges are labeled based on the distance measure. The algorithm seeks to improve uppon the classic $\mathrm{kNN}$ method by using information present in indirect neigbors. PGBCF achieves this by propagating probabilistic predictions along the edges of the user graph.

The rest of this paper is organized as follows. We start with a discussion of related approaches in Section 2. Next, we discuss variations on the Manhattan distance measure and propose the probabilistic representation in Section 3. Section 4 introduces probabilistic graph-based collaborative filtering. We present experiments comparing these approaches for various datasets and sparsity levels in Section 5. Finally, Section 6 states the main conclusions and lists opportunities for further research.

\section{Related Work}

A number of different general collaborative filtering techniques are described and compared in [1], including the nearest neighbor approach [10] which we will use to evaluate our proposed distance measure.

Methods specifically designed to alleviate the sparsity and cold start problem usually consist of a hybrid method between a collaborative filter and a contentbased recommender $[4,8,12]$. Our approach differs in that it remains a pure collaborative filter.

Our proposed method uses a probabilistic representation. Probabilistic representations have previously been used successfully in Bayesian approaches $[7,15]$.

A number of different graph based approaches have been proposed in the past as well, some of which were specifically designed to alleviate the sparsity problem.

Huang et al. [4] construct a bi-partite graph, containing users on one side and items on the other side. An edge between a user and an item is added when the user likes the item. An item is then ranked for a user based on the number of paths between that item and the user. Although the system gives good results, it is limited in use because it can only handle binary data.
A user either likes or dislikes an item, edges have no weight.

Papagelis [9] proposes another graph based algorithm in which the graph contains only users. The system uses only binary data: edges are weighted based on the number of co-ratings between two users.

In Alexandros [8] a method is described which exploits the transitive correlations between items. i.e. some items might be heavily correlated and thus a given rating for one item might tell us something about the rating for other items as well. The algorithm recommends the top- $k$ (transitively) correlated items. This can be seen as a hybrid approach to the sparsity problem.

Huang et al. [5] presents a graph based recommender system. Unlike our system, the system only recommends a number of items. No predictions are made for the other items. This system uses a two-layer graph, containing user-user, item-item and user-item edges. This graph is then searched for recommendable items.

Note that none of these graph based methods can be directly compared to our method either because they predict a ranking instead of an absolute value or because they can only handle binary data.

\section{Distance Measures and Representation}

In this section, we first discuss a popular method to deal with missing ratings when computing the similarity between two users' profiles. Next, we define a probabilistic profile representation, and define the notion of expected distance based on this representation, which leads to several natural ways to better handle missing ratings.

3.1 Distance Measures The similarity between two users' profiles (rows in the rating matrix) can be estimated in terms of a similarity measure or a distance measure. This paper considers the correlation coefficient (a similarity measure) and the Manhattan distance; both are frequently used in collaborative filtering [2]. To simplify notation, we first assume that no ratings are missing and then define how both measures are extended to handle missing ratings.

Given two user profiles $\mathbf{p}$ and $\mathbf{q}$, i.e., $n$-dimensional vectors of which the components are ratings taken from the set of possible ratings $R$ (e.g., $R=\{1,2,3,4,5\}$ ), Pearson correlation is defined as

$$
p c(\mathbf{p}, \mathbf{q})=\frac{\sum_{i}\left(p_{i}-\bar{p}\right)\left(q_{i}-\bar{q}\right)}{\sqrt{\sum_{i}\left(p_{i}-\bar{p}\right)^{2} \sum_{i}\left(q_{i}-\bar{q}\right)^{2}}},
$$

in which $\bar{u}$ denotes the average of $u$ 's components. The Manhattan distance is defined as 


$$
d_{M D}(\mathbf{p}, \mathbf{q})=\|\mathbf{p}-\mathbf{q}\|_{1}=\sum_{i=1}^{n}\left|p_{i}-q_{i}\right|,
$$

with $\|\cdot\|_{1}$ the 1 -norm.

In the presence of missing ratings, correlation is computed only over the components that are known in both $\mathbf{p}$ and $\mathbf{q}$. This approach does not apply to the Manhattan distance since the range of this measure depends on the number of components. Assuming the same distance along each dimension, this would result in a smaller value if more ratings are missing, which is not what we intend. Therefore, we compute the scaled Manhattan distance (MD-Scaled) instead, which is defined as

$d_{M D \text {-Scaled }}(\mathbf{p}, \mathbf{q})=\frac{n}{|K(\mathbf{p}) \cap K(\mathbf{q})|} \sum_{i \in(K(\mathbf{p}) \cap K(\mathbf{q}))}\left|p_{i}-q_{i}\right|$

with $K(\mathbf{u})=\left\{i \mid u_{i} \neq ?\right\}$ the set of indices for which $\mathbf{u}$ 's values are known. E.g., assume that $\mathbf{p}=(3,1, ?, 0, ?)$ and $\mathbf{q}=(1,2, ?, ?, ?)$, then $K(\mathbf{p})=\{1,2,4\}, K(\mathbf{q})=$ $\{1,2\}$ and $d_{M D \text {-Scaled }}(\mathbf{p}, \mathbf{q})=5 / 2(|3-1|+|1-2|)=5$.

As we will see in Section 5, the above measures's performance does not scale well with sparsity. If we have a sparsity of $50 \%$, i.e., the probability of a value being known is $p=0.5$, then the average fraction of overlap $|K(\mathbf{p}) \cap K(\mathbf{q})| / n$ between two vectors $\mathbf{p}$ and $\mathbf{q}$ is only $p^{2}=0.25$. Both correlation and $M D$-Scaled are undefined when $K(\mathbf{p}) \cap K(\mathbf{q})=\emptyset$, i.e., when the users do not have co-rated items.

3.2 Probabilistic Representation To address the aforementioned instability of the similarity and distance measures, we propose a probabilistic representation with probabilistic user profiles, in which crisp ratings are replaced by probability distributions over the domain of possible ratings. That is, we replace each vector component $p_{i}$ by its marginal probability distribution $\operatorname{Pr}\left(p_{i}\right)\left(\sum_{u \in R} \operatorname{Pr}\left(p_{i}=u\right)=1\right)$.

We can compute the expected Manhattan distance (EMD) between two probabilistic user profiles as

$$
\begin{aligned}
& d_{E M D}(\mathbf{p}, \mathbf{q}) \\
& =E\left[\|\mathbf{p}-\mathbf{q}\|_{1}\right] \\
& =\sum_{i=1}^{n} \sum_{u_{p} \in R} \sum_{u_{q} \in R}\left|u_{p}-u_{q}\right| \operatorname{Pr}\left(p_{i}=u_{p}\right) \operatorname{Pr}\left(q_{i}=u_{q}\right) .
\end{aligned}
$$

We assume here that the random variables corresponding to different ratings are independent.

For known ratings $p_{i}=p \neq ?, \operatorname{Pr}\left(p_{i}\right)$ is defined as

$$
\operatorname{Pr}\left(p_{i}=u\right)=\left\{\begin{array}{ll}
1 & \text { if } u=p \\
0 & \text { if } u \neq p
\end{array} .\right.
$$

The distribution for vector components that correspond to unknown ratings will be different. We will now consider several ways to define a distribution for this case.

Distribution for Unknown Ratings Which distribution should we use to calculate the EMD if a rating for an item is unknown? We consider the following two possibilities.

- Assume that the item's rating follows the uniform distribution $\operatorname{Pr}\left(p_{i}=u\right)=1 /|R|$, with $u \in R$. We call the expected Manhattan distance computed based on this assumption the expected Manhattan distance - uniform or EMD-Uni for short.

- Suppose that the rating for item $i$ for user $j$ is unknown. Assuming that user $j$ rates similar to all other users, we can use the global distribution of all known ratings for that particular item $i$ instead of the uniform distribution. We will refer to the resulting distance as the expected Manhattan distance - global distribution or EMD-GD for short.

Consider the first option. If both users did not rate a given item, then the term in the formula for $d_{E M D}$ that corresponds to this item only depends on the rating domain $R$. It does not depend on the user or on the actual item. For example, if $R=\{1, \ldots, r\}$ then this value is the constant $c=\sum_{u_{p}} \sum_{u_{q}} \mid u_{p}-$ $u_{q}\left|\operatorname{Pr}\left(p_{i}=u_{p}\right) \operatorname{Pr}\left(q_{i}=u_{q}\right)=\frac{1}{r^{2}} \sum_{u_{p}} \sum_{u_{q}}\right| u_{p}-u_{q} \mid=$ $r\left(r^{2}-1\right) /\left(3 r^{2}\right)$. This value can be seen as a penalty that is added to the Manhattan distance for each item not rated by both users. This motivates the following distance measure definition: compute the Manhattan distance over the items that are known for both users and add for each item that is not rated by both users the penalty $c$. We call this distance measure Manhattan distance - penalty or MD-Penalty. The main difference between EMD-Uni and EMD-Penalty lies in the items that are rated by only one of the users: for these items $M D$-Penalty also assigns a penalty of $c$, while $E M D$ Uni computes the expected distance based on the given distributions. $M D$-Penalty can be computed a factor $|R|$ faster than EMD-Uni and EMD-GD.

\section{Probabilistic Graph-based collaborative filtering}

In this section we will explain how a graph can be used as the basis for collaborative filtering methods.

4.1 The User Graph We represent the data by a so called user graph $G=(V, E)$. Each node $\mathbf{v} \in$ $V$ represents a user. If a distance $d$ between two users/nodes can be computed, a weighted edge $e \in E$ 
$\overline{\text { Algorithm } 1 \mathrm{kNN} \text { predicts the missing ratings in the }}$ ratings in a matrix $A$. $E$ is the set of edges defining the user graph, $w$ is the edge weight function, and $k$ is the number of neighbors to consider.

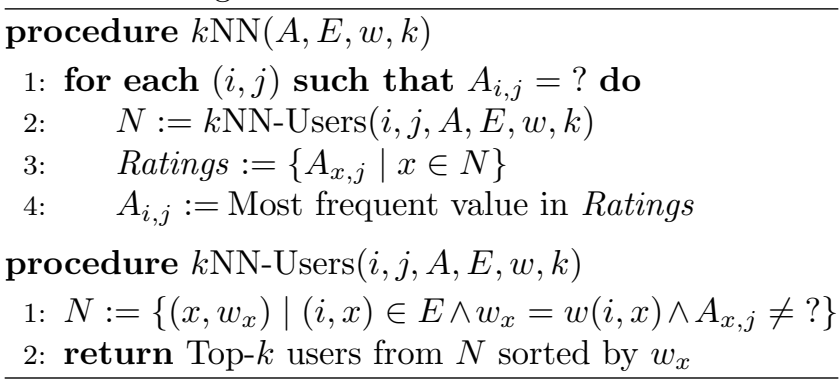

between those nodes is added to the graph. The weight $w$ of that edge is inversely proportional to the distance between the nodes it connects:

$$
w_{e\left(\mathbf{v}_{\mathbf{1}}, \mathbf{v}_{\mathbf{2}}\right)}=\frac{1}{d\left(\mathbf{v}_{\mathbf{1}}, \mathbf{v}_{\mathbf{2}}\right)}
$$

The graph will not contain edges between users without co-rated items when using Pearson Correlation or MD-Scaled, When using EMD or MD-Penalty, this procedure will result in a fully connected graph.

To use the graph in a collaborative filtering method, we have to complete information in each node, based on the information present in the connected nodes.

We start by translating the classic nearest neighbor approach to a graph based method. This method is then extended to make explicit use of the graph structure.

\subsection{Nearest Neighbor Graph Completion Sup-} pose we have a node $\mathbf{v}=(3,-1, ?, 0, ?)$. Our goal then consists of predicting the missing values $v_{3}$ and $v_{4}$. We can use the constructed graph to make these predictions. Since a higher edge-weight means a higher similarity, that is a closer neighbor, the missing values are more likely to be equal to the corresponding values of connected nodes for which the edge-weight of the connecting edge is high. A missing value can then be predicted as follows: select the $k$ connected nodes with the highest edge weights, for which the value to be predicted is known, and take the average of those known values. This corresponds to the $k$ nearest neighbors approach [10].

While this method gives good results in most cases [1], it suffers from the so called Cold Start problem [13]. Users who only rated a few items will be given bad ratings, and new items, only rated by a few users, will rarely be recommended.

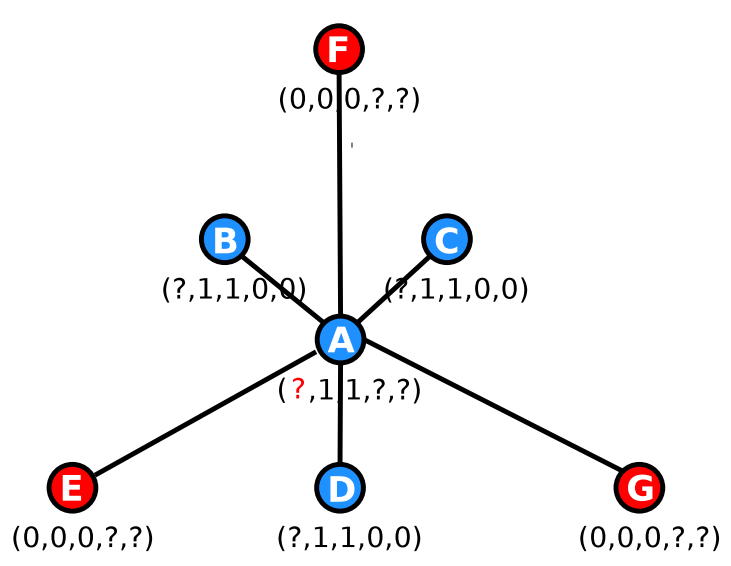

Figure 1: The sparsity problem. Red users have a known rating for item 1. Although user $\mathrm{A}$ has 3 close neigbors (B,C and D), none of them has a known rating for item 1. To make a prediction for item 1, user A has to rely on distant users E,F and G, resulting in a not so accurate prediction. Edge lengths are proportional to the distance between users.

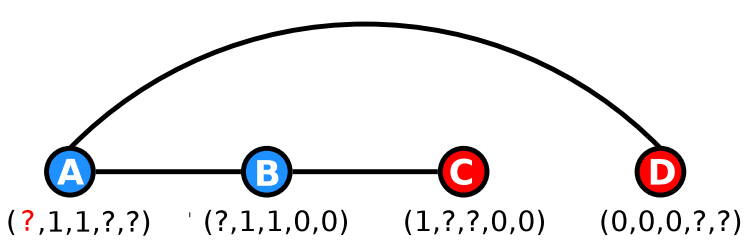

Figure 2: Using indirect neigbors. Users $C$ and $D$ gave a rating to item 1 . But only $D$ is a (distant) neighbor of user $A$. 


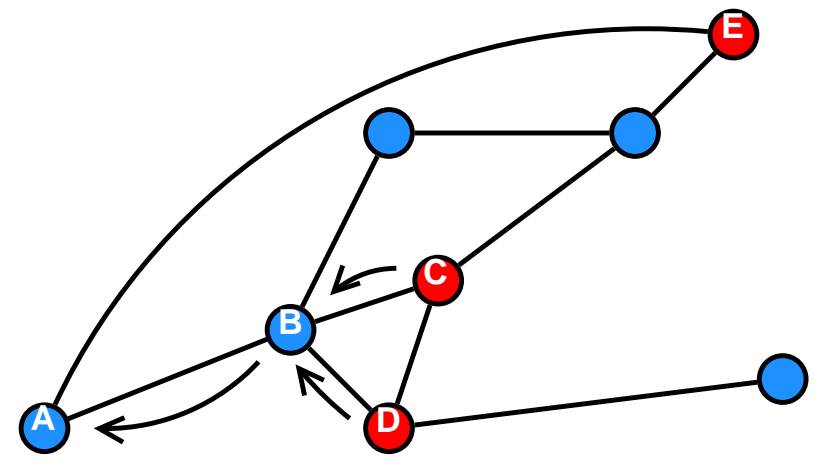

Figure 3: A user-graph. Each node represents a user in the dataset. When a distance between two users can be computed, a weighted edge is placed between those users. The arc lengths correspond to distances. Known values (in $C, D$ and $E$ ) can be propagated, eg from $C$ and $D$ to $B$ and to $A$ subsequently. Without propagation, the predicted value for user $A$ would be based solely on user $E$, resulting in a bad prediction due to the large distance between $A$ and $E$.

4.3 PGCF To alleviate the cold start and the sparsity problem we propose to use close but indirect neighbors instead of almost unrelated neighbors. This is illustrated in figures 3 and 2 . In figure 2 the goal is to predict a rating for item 1, the red question mark, for user $A$. The only direct neighbor who rated this item too is user $D$. The resulting prediction would be 0 since user $D$ gave the first item a rating of zero. If we take a closer look at the common ratings between users $A$ and $D$, we notice that they gave completely opposite ratings. User $A$ rated items 2 and 3 with a 1 while $D$ gave them both a 0 . It is therefore unlikely that the prediction we made was very accurate. If we look at the common ratings between $A$ and $B$, the common ratings are identical and we can assume that they would rate other items similarly as well. The same is true for $B$ and $C$. We can thus argue that $B$ would rate item 1 with a 1 since user $C$ did so, and if $B$ would rate item 1 with a 1 , user $A$ would do so too. By making the prediction for user $B$ first and subsequently using this prediction to predict item 1 for user $A$, we can largely avoid the sparsity problem and obtain a more accurate prediction.

This method of propagating predictions will form the basis of our graph based collaborative filtering algorithm. One problem with this approach is the
Algorithm 2 PGBCF predicts the missing ratings in the ratings in a matrix $A$. $E$ is the set of edges defining the user graph, $w$ is the edge weight function, $k$ is the number of neighbors to consider, and $R$ is the rating domain. See Alg. 1 for $k \mathrm{NN}-U$ sers.

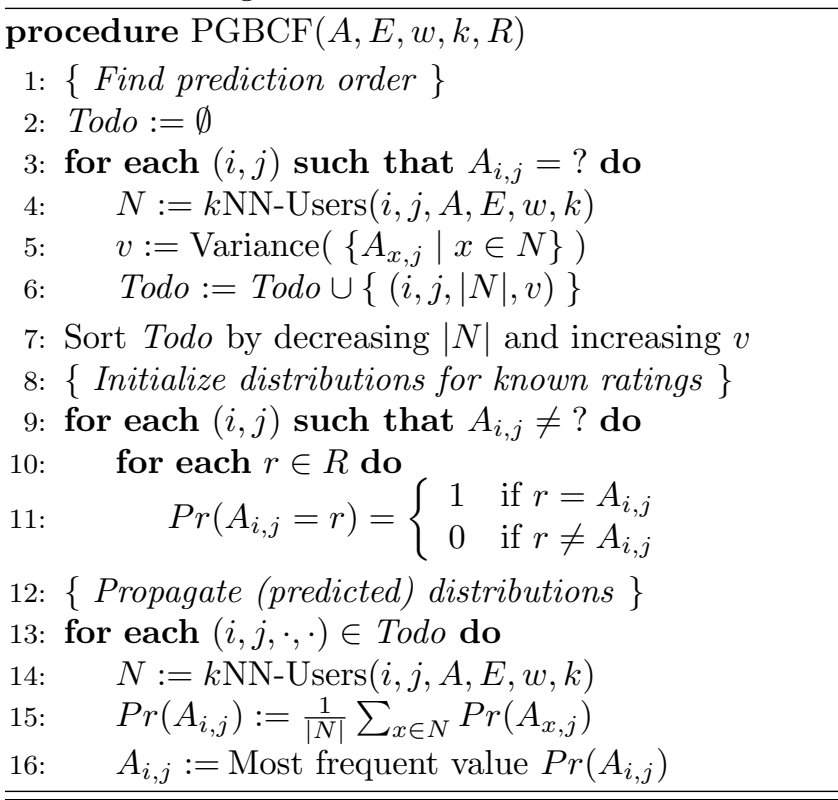

following. Making predictions and regarding them as known values is prone to divergence. Errors would be propagated and start to accumulate. To remedy this we have to take two measures:

- make the most certain prediction first

- take the uncertainty of previous predictions into account when using them for other predictions

The Prediction Order Our goal is to fill in first the predictions we can make most confidently. To this end, we need a way to determine the confidence we have in a prediction. Suppose we make a prediction based on $k$ neighbors and all of those neighbors rated the item for which we are predicting a rating exactly the same. This will give us a prediction with a maximum confidence. If, on the other hand, all the neighbors rate the item differently, we will not be able to make a confident prediction.

The variance of the neighbor-ratings used to make the prediction gives us thus a measure of confidence of the prediction. A low variance tells us the prediction is most likely correct. A high variance tells us we have low confidence in the prediction.

We can now use the variance to order the sequence in which we will fill in predictions in the graph.

Propagating Uncertainty Suppose our users can only rate an item positive or negative and we are making 
a prediction for some item for user $A$ based on the three nearest neighbors. Two out of three neigbors rated the item positively and one neighbor gave the item a negative rating. In this case, we would predict that user $A$ would rate the item positive but we would not be very confident in this prediction. Only $2 / 3$ neighbors gave a positive rating, i.e. we would assign a confidence level of $2 / 3$ to the prediction.

In our algorithm, predicted values are used for subsequent predictions. For example: when making a prediction for user $B$, this user might have user $A$ as one of its neigbors. If the neighbors of $B$ (other than $A$ ) all rate the item positively, and since our prediction for $A$ was positive, the prediction for $B$ would be positive too. Assigning a confidence value to this prediction is now a little harder. If we regard the predicted value for $A$ as a known value, all of $B$ 's neighbors would have rated the item positively and the resulting confidence value would be 1 . Doing so disregards the fact that we were not completely confident in our prediction for user $A$.

As a solution to this shortcoming, we propose to store the distribution of ratings among the neigbors instead of the prediction resulting from this distribution, i.e. instead of storing a positive prediction for user $A$, we would store $(\oplus 2 / 3, \ominus 1 / 3)$. When making the prediction for user $B$, we use this distribution instead of the prediction. This is accomplished by splitting $A$ 's vote in the prediction for $B$ according to the distribution. i.e. if $B$ has two neigbors with a positive vote and the distribution of user $A$, we would get the following predicted distribution for user $B:(\oplus=$ $\frac{1+1+2 / 3}{3}=\frac{8}{9}, \ominus=\frac{1 / 3}{3}=\frac{1}{9}$ ) or a positive prediction for $B$ with a confidence of $8 / 9$. The fact that we were not completely confident in our prediction for $A$ is now taken into account when making the prediction for $B$ and is reflected in the confidence we have in the prediction for user $B$. A second benefit from this method is that a low confidence prediction has less influence on a subsequent prediction as its vote is split up more evenly.

As our algorithm works on non-binary data, the method described above is extended to discrete distributions. E.g. suppose we have ratings between 1 and 5 and 5 neighbors rating $3,4,3,5$ and 2 respectively, we would get the following distribution $\operatorname{Pr}=\left(0, \frac{1}{5}, \frac{2}{5}, \frac{1}{5}, \frac{1}{5}\right)$ Since a rating could be the result of a prediction, it's possible that the rating is represented as a distribution. A distribution $D$, based on the distributions $\operatorname{Pr}^{i}$ of $n$ neighbors is then calculated as follows:

$$
\operatorname{Pr}_{j}=\sum_{i=1 . . n} D_{j}^{i} / n
$$

The final prediction is made by taking the median of this distribution. To compare the confidence we have in a prediction we can use the variance of the distribution. The lower the variance, the more confident we are in a prediction. i.e. $D=(0,1,0,0,0)$ has a variance of 0 , all neighbors gave the same rating (2 in this case) and thus the prediction would be accurate.

\section{Experimental results}

We have run a number of experiments in order to evaluate the performance of the proposed distance measures and graph based algorithms. The experiments are run on the well known MovieLens [10] dataset, a subset of this dataset consisting of the top 100 most rated movies and the top 100 users who gave the most ratings as used in [14]. Our second dataset is the Jester joke database, consisting of a number of rated jokes. The details of these datasets can be found in table 1 and figure 4 shows the distribution of the number of rated items by the users.

Table 1: Description of the MovieLens100k, the top100 subset and the Jester dataset.

\begin{tabular}{c|ccc}
\hline & ML-100K & ML-Top100 & Jester \\
\hline \# users & 943 & 100 & 73,421 \\
\# items & 1682 & 100 & 100 \\
\# rated items & 100,000 & 2914 & 4.1 Million \\
sparsity & 0.93 & 0.70 & 0.44
\end{tabular}

To evaluate the performance, the dataset is split in 5 disjoint train/test splits, with $80 \%$ of the data in each training set. Performance is measured using the mean average error (MAE). We will discuss the performance under varying sparsity levels and analyze in depth the performance of the algorithm for a new user, i.e., the effect of the cold start performance.

5.1 Distance Measure Evaluation Figure 5 shows the cross-validated mean absolute error as a function of training set sparsity for nearest neighbor (Algorithm 1) in combination with the distance measures discussed in Section 3. These include the Pearson correlation (PC), scaled Manhattan distance (MD-Scaled), Manhattan distance with penalty (MD-Penalty), and the expected Manhattan distance based on the global (EMD-GD) and uniform (EMD-Uni) distribution. The figure also indicates the default MAE, which corresponds to always predicting the overall median of the rankings in the dataset. Other methods of alleviating the sparsity problem are not considered in this comparison because their performance has little influence on the usefulness of our proposed distance measure. For example, using a hybrid method [12] to alleviate the sparsity problem 

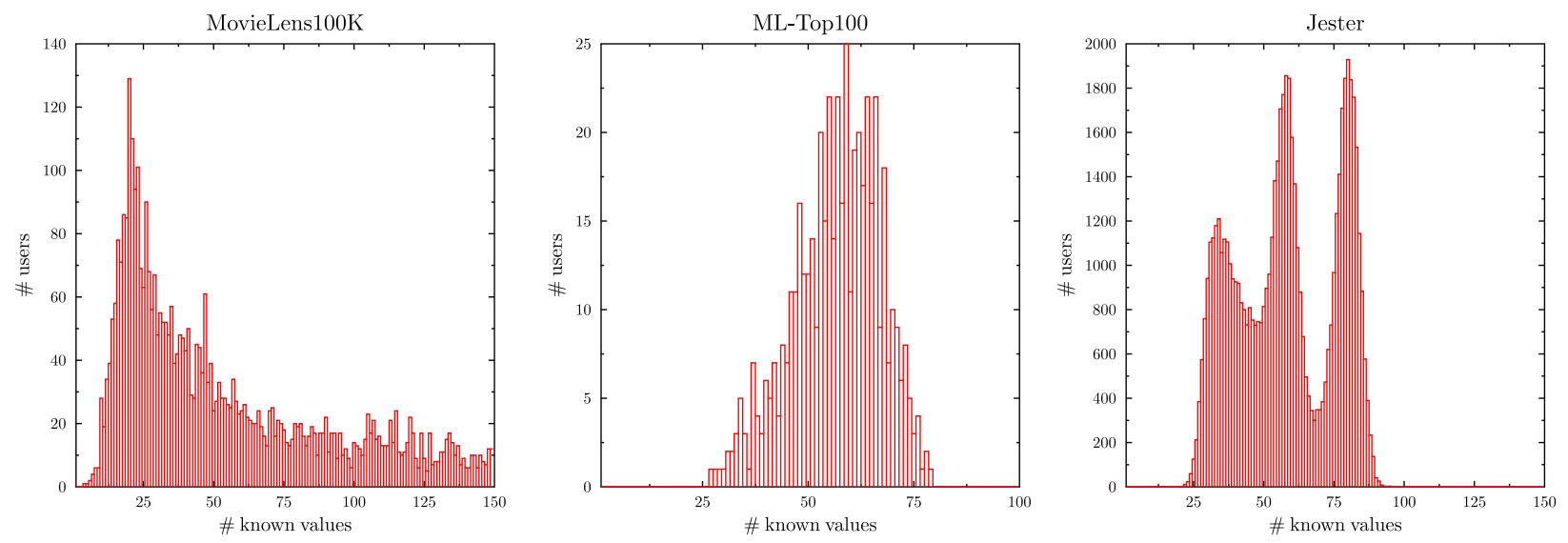

Figure 4: Number of users for each number of known ratings.

could very well be combined with our distance measures. Note that the default MAE is not useful in practice as it can not be used to rank items.

$\mathrm{PC}$, which is frequently used in collaborative filtering, and EMD-Uni are consistently outperformed by both MD-Penalty and EMD-GD across all datasets and sparsity levels. MD-Scaled performs best or second-best for low dataset sparsity. If sparsity increases, however, its performance significantly deteriorates; MD-Scaled is the worst performing distance measure on sparse datasets. The reason is that this distance measure becomes unstable if the overlap between the users' profiles is too small (Section 3.1). MD-Penalty and EMD-GD perform well overall. They have similar performance on MovieLens-Top100. The results on Jester, and to a lesser extent also those on MovieLens-100K, show that MD-Penalty works best for low sparsity levels, while EMD-GD is better for high sparsity levels. Based on these observations, we recommend EMD-GD for sparse datasets and MD-Penalty for less sparse datasets.

Recall that MD-Penalty adds a fixed penalty to the Manhattan distance for each item that is not rated by both users. As a result, MD-Penalty explicitly takes the number of common ratings into account. A lower number of co-rated items yields a higher distance. One might argue that users with a high number of common ratings are more likely to have similar taste, irrespectively of the ratings given. This is reflected in the MD-Penalty measure.

5.2 Performance of PGBCF Figure 6 shows the performance of probabilistic graph based collaborative filtering (PGBCF) compared to that of $k \mathrm{NN}$. Both use MD-Scaled as distance measure. Comparing to the graph based algorithms described in section 2 is not possible due to them only working on binary datasets or directly predicting a rank.

PGBCF performs comparable to $k \mathrm{NN}$ in most of the experiments. We expect PGBCF to be most useful on sparse datasets. If the data is not sufficiently sparse, then propagating predictions is less useful and may decrease accuracy (this is known as the over activation effect [4]). This hypothesis is confirmed by the results: on MovieLens-100K, PGBCF outperforms $k \mathrm{NN}$ on sparse datasets. The same is true for Jester. Therefore, PGBCF should be considered for predicting ratings on sparse datasets.

PGBCF's computational cost is about twice that of $k \mathrm{NN}$ (ignoring the time required for sorting the unknown ratings). So, the overhead of propagating predictions is relatively small. This is only the case if the goal is to compute item ratings for all users. If we are interested in making predictions only for one user, then this can be easily accomplished with $k \mathrm{NN}$, but not with PGBCF because it makes predictions for all users in a pre-determined order.

Combining the MD-Penalty distance measure with the graph based method gives a small additional improvement on the MovieLens dataset for high sparsity levels (see figure 7). When the dataset gets less sparse, kNN-MD-Scaled outperforms the combined method. This is a result of PGBCF performing slightly worse than kNN-MD-Penalty on the not so sparse datasets. For the Jester dataset, the combined method performs close to kNN-MD-Penalty, except for when PGBCF outperforms kNN-MD-Penalty, in which case the combined method also outperforms kNN-MD-Penalty.

5.3 Robustness Against Cold Start Problem To analyze the robustness of the algorithms with regard to the cold start problem, we measure the MAE over users of which the number of rated items lies in a given 

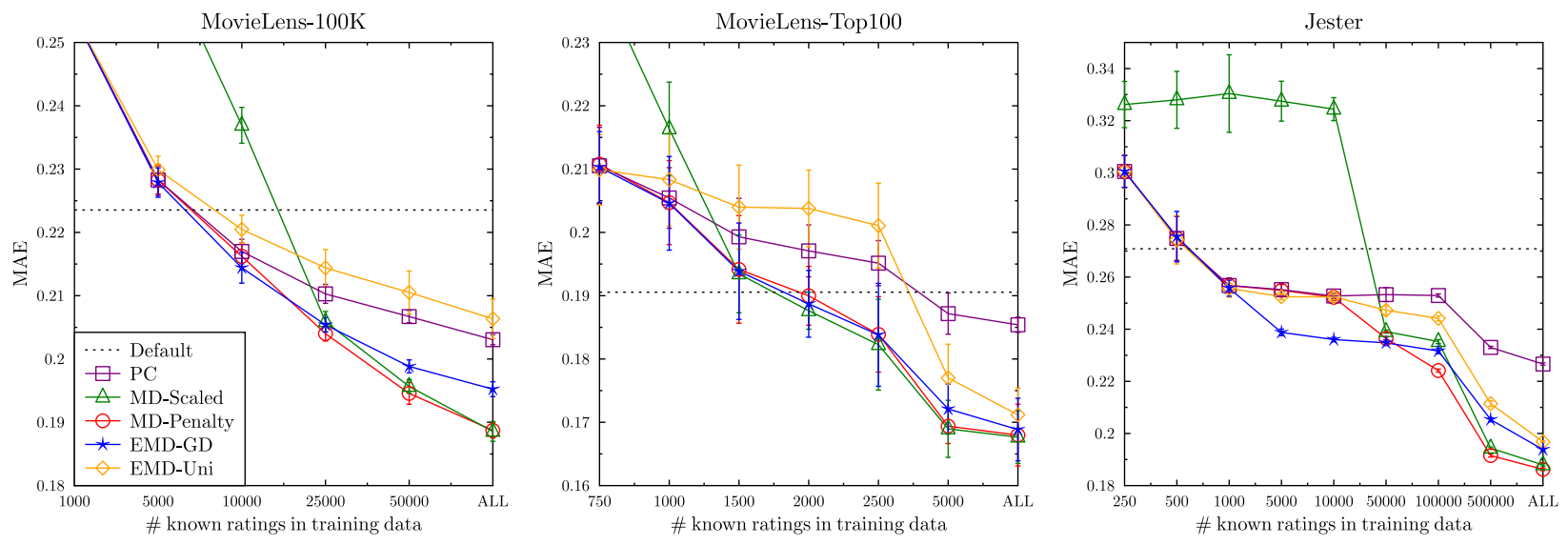

Figure 5: Cross-validated MAE versus dataset sparsity for 10NN in combination with different distance measures
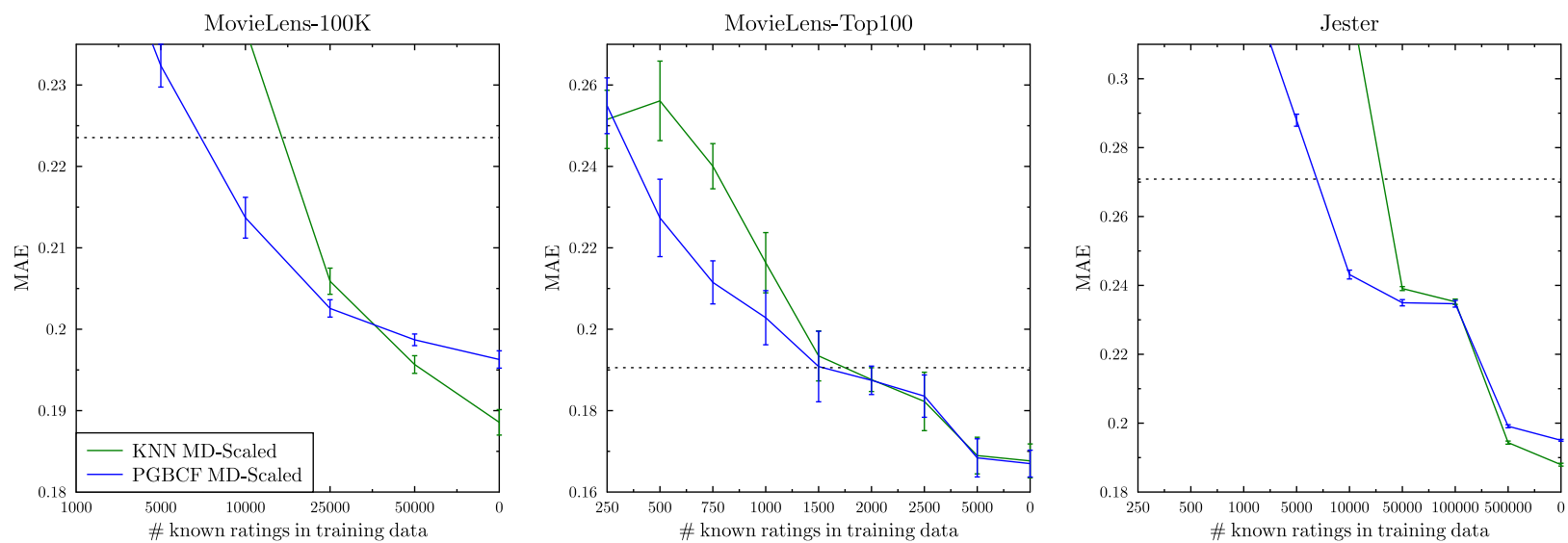

Figure 6: Cross-validated MAE of PGBCF and $k$ NN, both using MD-Scaled, across various sparsity levels. We use the original scaled distance measure to study the performance using the graph based method.

interval. For example, the MAE of all users who rated only 2-3 items shows how the algorithms perform on new users. Figure 8 shows these results for different intervals. We only show results for MovieLens-100K. For the other datasets, there are no users who rated only this few items (See histograms in Figure 4).

The results clearly show that the new distance measure outperforms the commonly used Pearson correlation. Although the variance on the results is high, we observe that PGBCF performs better than $k \mathrm{NN}$ for users who rated a small number of items. Only for users who rated only 2 or 3 items, PGBCF drops below $k \mathrm{NN}$.

\section{Conclusions and Further Work}

This paper's goal was to address the sparsity and cold start problems by switching to a probabilistic representation of the rankings. We first proposed a number of variations on the Manhattan distance that make use of this representation (EMD-GD and EMD-Uni) or that are inspired by it (MD-Penalty). Next, we employed this representation in probabilistic graph based collaborative filtering $(\mathrm{PGBCF})$, an algorithm that propagates probabilistic ranking predictions through the user graph.

Experiments show that the new distance measures (in particular, MD-Penalty and EMD-GD) consistently outperform common similarity and distance measures such as Pearson correlation and the scaled Manhattan 

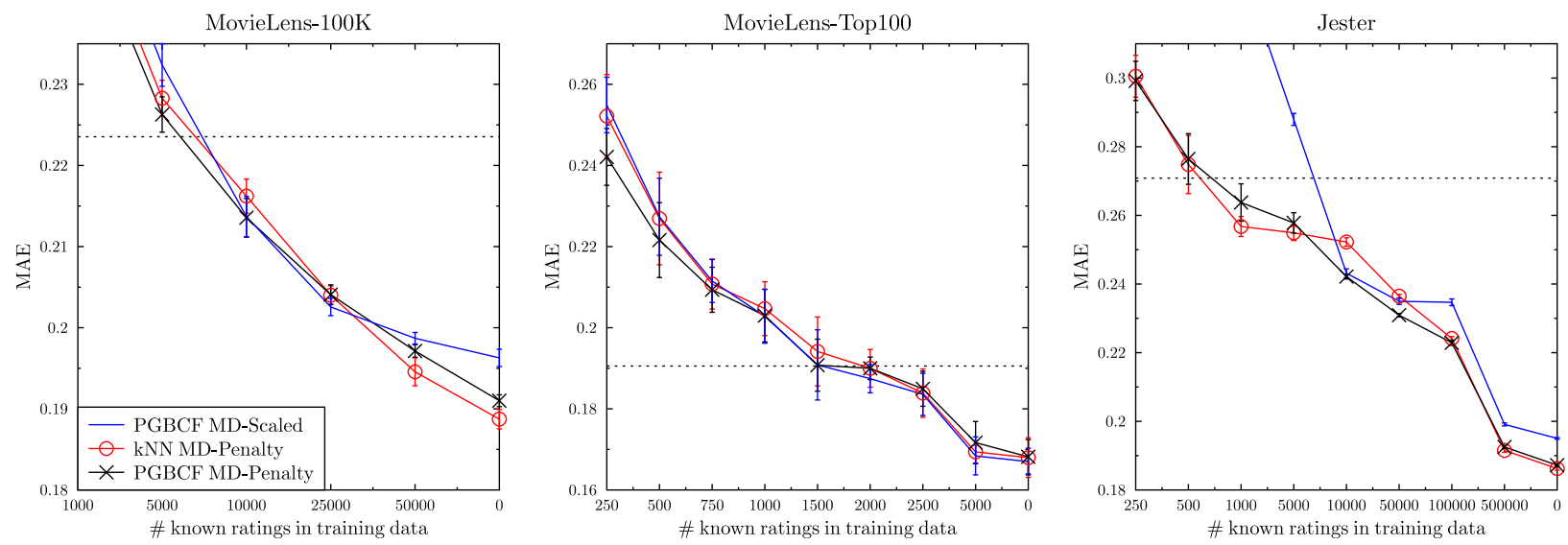

Figure 7: Combing our distance measure and our graph based approach.

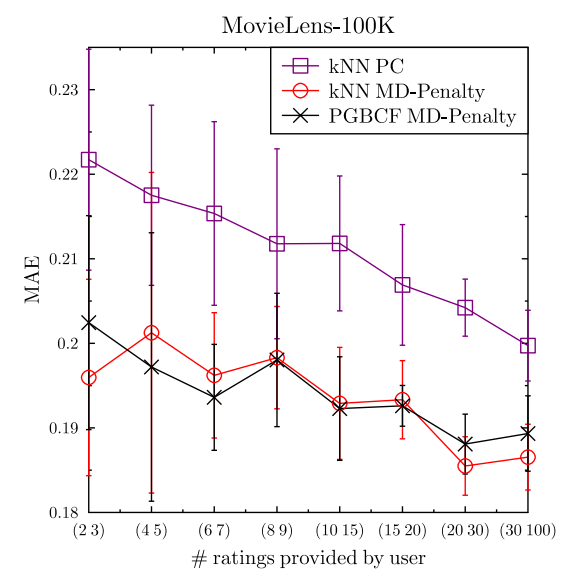

Figure 8: 5-fold Cross-validated average error across users who rated a given number of items distance. They should definitely be considered in practical applications. Further results show that PGBCF may outperform nearest neighbor on sparse datasets or on new users who rated only a few items.

\section{References}

[1] Gediminas Adomavicius and Alexander Tuzhilin. Toward the next generation of recommender systems: A survey of the state-of-the-art and possible extensions. IEEE Transactions on Knowledge and Data Engineering, 17(6):734-749, June 2005.

[2] Laurent Candillier, Frank Meyer, and Françoise Fessant. Designing specific weighted similarity measures to improve collaborative filtering systems. In ICDM '08: Proceedings of the 8th industrial conference on Advances in Data Mining, pages 242-255, Berlin, Heidelberg, 2008. Springer-Verlag.

[3] Thomas Hofmann. Latent semantic models for collaborative filtering. ACM Transactions on Information Systems, 22(1):89-115, 2004.

[4] Zan Huang, Hsinchun Chen, and Daniel Zeng. Applying associative retrieval techniques to alleviate the sparsity problem in collaborative filtering. $A C M$ Trans. Inf. Syst., 22(1):116-142, January 2004.

[5] Zan Huang, Wingyan Chung, Thian-Huat Ong, and Hsinchun Chen. A graph-based recommender system for digital library. In JCDL '02: Proceedings of the 2nd ACM/IEEE-CS joint conference on Digital libraries, pages 65-73, New York, NY, USA, 2002. ACM.

[6] Zan Huang, D. Zeng, and Hsinchun Chen. A comparison of collaborative-filtering recommendation algorithms for e-commerce. Intelligent Systems, IEEE, 22(5):68-78, 2007.

[7] Koji Miyahara and Michael J. Pazzani. Collaborative filtering with the simple bayesian classifier. In In: Pro- 
ceedings of the 6th Pacific Rim International Conference on Artificial Intelligence, pages 679-689, 2000.

[8] Alexandros Nanopoulos. Collaborative filtering based on transitive correlations between items. Advances in Information Retrieval, 2007.

[9] M. Papagelis, D. Plexousakis, and T. Kutsuras. Alleviating the sparsity problem of collaborative filtering using trust inferences. In P. Herrmann, editor, iTrust, pages 224-239. Springer-Verlag Berlin Heidelberg, 2005.

[10] Paul Resnick, Neophytos Iacovou, Mitesh Suchak, Peter Bergstrom, and John Riedl. Grouplens: An open architecture for collaborative filtering of netnews. pages 175-186. ACM Press, 1994.

[11] Badrul M. Sarwar, George Karypis, Joseph A. Konstan, and John T. Riedl. Application of dimensionality reduction in recommender system - a case study. In In ACM WebKDD Workshop, 2000.

[12] Andrew I. Schein, Alexandrin Popescul, Lyle H., Rin Popescul, Lyle H. Ungar, and David M. Pennock. Methods and metrics for cold-start recommendations. In In Proceedings of the 25th Annual International ACM SIGIR Conference on Research and Development in Information Retrieval, pages 253-260. ACM Press, 2002.

[13] Andrew I. Schein, Alexandrin Popescul, Lyle H. Ungar, and David M. Pennock. Methods and metrics for coldstart recommendations. In SIGIR '02: Proceedings of the 25th annual international ACM SIGIR conference on Research and development in information retrieval, pages 253-260, New York, NY, USA, 2002. ACM.

[14] Nathan Srebro, Jason D. M. Rennie, and Tommi S. Jaakkola. Maximum-margin matrix factorization. In Advances in Neural Information Processing Systems 17, pages 1329-1336, 2005.

[15] Xiaoyuan Su and Taghi M. Khoshgoftaar. Collaborative filtering for multi-class data using belief nets algorithms. In ICTAI '06: Proceedings of the 18th IEEE International Conference on Tools with Artificial Intelligence, pages 497-504, Washington, DC, USA, 2006. IEEE Computer Society. 